\title{
Covid-19 e suas consequências na retomada da prática do exercício físico
}

\author{
COVID-19 and its consequences in resuming the practice of physical exercise \\ COVID-19 y sus consecuencias al reanudar la práctica de ejercicio físico
}

Ozimikaele Silva Ricarte

Universidade Estadual da Paraiba, Brasil

ozimikaele.ricarte@aluno.uepb.edu.br

(D) https://orcid.org/0000-0002-2697-4052

\author{
Marlene Salvina Fernandes Costa \\ Universidade Federal de Pernambuco, Brasil \\ marlenesfc@hotmail.com
}

(DD https://orcid.org/0000-0002-2188-6686

\author{
Pedro Pinheiro Paes \\ Universidade Federal de Pernambuco, Brasil \\ pppaes@ufpe.br \\ (iD https://orcid.org/0000-0002-6765-5672
}

\author{
Walmir Romário dos Santos \\ Universidade de São Paulo, Brasil \\ walmir@alumni.usp.br \\ (D) https://orcid.org/0000-0001-5558-3109
}

\author{
Wlaldemir Roberto dos Santos \\ Universidade Estadual da Paraiba, Brasil \\ wlaldemir@hotmail.com \\ iD https://orcid.org/0000-0001-5706-2243
}

\section{Resumo:}

O objetivo do presente estudo foi discutir e analisar as consequências enfrentadas no retorno há prática de atividade física do indivíduo acometido pela COVID-19. Realizou-se uma pesquisa descritiva com delineamento exploratório do tipo survey com 34 participantes, acometidos pela COVID-19 e com histórico prévio de treinamento físico. Os instrumentos de pesquisa foram construídos a partir de dois blocos independentes: a) caracterização dos participantes e; b) aplicação do SF-36. Os dados não apresentaram normalidade pelo teste de Shapiro-Wilk e homogeneidade pelo teste de Levene's. Os resultados foram apresentados a partir de uma análise descritiva. O teste Qui quadrado mostrou associação significativa entre as variáveis fraqueza ou fadiga muscular e dispneia $(\mathrm{p}<0,05)$. Foi observado que os participantes, em especial as mulheres, relataram perda de desempenho. Por se tratar de uma condição de saúde pouco conhecida, análises mais aprofundadas devem ser realizadas para evidenciar as consequências do COVID-19, principalmente em estudos longitudinais.

Palavras-ChaVe: COVID-19, Condições de saúde, Exercício físico, Isolamento social, Pandemia.

\begin{abstract}
:
The aim of this study was to discuss and analyze the consequences faced in the return to physical activity of the individuals affected by COVID-19. Descriptive research with an exploratory survey design was carried out with 34 participants, all of them affected by COVID-19 and with a previous history of physical training. The research instruments were built from two independent blocks:
\end{abstract}


a) characterization of the participants and; b) application of SF-36. The data did not show normality according to the ShapiroWilk test and homogeneity according to the Levene's test. The results were presented from a descriptive analysis. The Chi-square test showed a significant association between the variables muscle weakness or fatigue and dyspnea $(\mathrm{p}<0.05)$. It was observed that the participants, especially women, reported performance loss. As COVID-19 is a little-known health condition, deeper analyzes should be carried out to highlight its consequences, especially in longitudinal studies.

KEYWORDS: COVID-19, Health conditions, Physical exercise, Social isolation, Pandemic.

\section{ReSUMEN:}

El objetivo del presente estudio fue discutir y analizar las consecuencias que enfrenta cuando el individuo afectado por COVID-19 regresa a la práctica de actividad física. Se realizó una investigación descriptiva con un diseño tipo encuesta exploratoria con 34 participantes, afectados por COVID-19 y con antecedentes de entrenamiento físico. Los instrumentos de investigación se construyeron a partir de dos bloques independientes: a) caracterización de los participantes y; b) aplicación del SF-36. Los datos no mostraron normalidad por la prueba de Shapiro-Wilk y homogeneidad por la prueba de Levene. Los resultados se presentaron a partir de un análisis descriptivo. La prueba de Chi-cuadrado mostró asociación significativa entre las variables debilidad o fatiga muscular y disnea $(\mathrm{p}<0.05)$. Se observó que los participantes, especialmente las mujeres, informaron pérdida de rendimiento. Como se trata de una condición de salud poco conocida, se deben realizar más análisis para resaltar las consecuencias del COVID-19, especialmente en estudios longitudinales.

Palabras Clave: COVID-19, Condiciones de salud, Ejercicio físico, Aislamiento social, Pandemia.

\section{INTRODUÇÃO}

O SARS-CoV-2, coronavírus relacionado à síndrome respiratória aguda grave (COVID-19), foi detectado pela primeira vez na China em dezembro de 2019, se alastrando rapidamente pelo mundo e decretado como uma pandemia em março de 2020 (OMS, 2020). No Brasil, a maioria das pessoas com COVID-19 (80\%) podem ser assintomáticas ou ter sintomas leves, sendo que cerca de $20 \%$ dos casos podem requerer atendimento hospitalar com quadros respiratórios graves e 2,8\% evoluir para o óbito (BRASIL, 2021), principalmente idosos e pessoas com comorbidades como cardiopatias, pneumopatias, doenças renais ou alterações metabólicas (OPAS, 2020). Segundo a Organização Mundial da Saúde, já chegamos a 165.158.285 de casos confirmados da COVID-19, incluindo 3.425.017 mortes. No Brasil conforme o boletim epidemiológico divulgado pelo Ministério da Saúde atualizado no dia 21/05/2021 temos 15.812.055 casos confirmados e 441,691 óbitos (WHO, 2021).

Além do risco de óbito, o COVID-19 impacta diretamente a qualidade de vida das pessoas que foram acometidas, devido as complicações da fase aguda da doença e possíveis sequelas que tem afetado boa parte dos contaminados, podendo impactar nos aspectos físicos, funcionais e emocionais (Huang et al., 2021; Iser et al., 2020).

O Colégio Americano de Medicina do Esporte (ACSM, 2020) divulgou um guia em que sugere que a atividade física de intensidade moderada deva ser mantida em função do das complicações do COVID-19, salientando a importância para a saúde de cada minuto fisicamente ativo. Para eles, atividade física é extremamente relevante para uma boa saúde, ajudando a prevenir e/ou tratar possíveis condições de saúde física e mental, melhorando o funcionamento de vários sistemas fisiológicos, promovendo uma resposta positiva do sistema imunológico.

Devido ao curto espaço de tempo, estudos sobre os efeitos do exercício diretamente sobre os pacientes com COVID-19 ainda não foram descritos, porém sugere-se respostas parecidas com demais infecções virais, sobre a imunidade, inflamação e infecções, principalmente nas vias respiratórias, sugerindo menor risco de agravamento da doença caso seja exposto ao vírus (Luan et al., 2019). Entretanto, tem sido descrito que o exercício físico, além de atuar como um agente potencializado na fase aguda da doença, pode contribuir diretamente com as complicações decorrentes das sequelas apresentadas, sendo que após o período agudo da 
doença a retomada da prática do exercício físico deve acontecer o mais breve possível (Gomes e Paula, 2020; ACSM, 2020).

Todavia, algumas sequelas podem causar desconforto e reduzir o desempenho físico na volta ao exercício, podendo impactar na adesão dos praticantes. Isso ocorre devido a resposta inflamatória excessiva ocasionada pela infecção do COVID-19, potencializando as atividades catabólicas, podendo aumentar os níveis de fadiga e fraqueza muscular, contribuindo para uma resposta muscular hipotrófica (Huang et al., 2021), além dos danos ao sistema respiratórios, onde os pacientes podem apresentar quadros dispneicos (Iser et al., 2020).

Deste modo, devido ao impacto do COVID-19 na população mundial, as sequelas que a infecção pode ocasionar, a importância da prática do exercício físico após a contaminação, as consequências funcionais que a sequela pode apresentar e impactar na saúde, o presente estudo teve como objetivo verificar a qualidade de vida e as dificuldades apresentadas na retomada do exercício após o COVID-19, na perspectiva de ter maiores parâmetros científicos para o melhor enfrentamento da doença.

\section{METODOLOGIA}

\section{Desenho do estudo}

Trata-se de uma pesquisa descritiva com delineamento exploratória do tipo survey (Thomas, Nelson e Silverman, 2012), realizado via Google Formulário.

\section{Participantes}

Participaram do estudo 34 sujeitos (58,8\% mulheres), sendo que todos foram acometidos pelo COVID-19, praticavam exercício físico há pelo menos 6 meses com frequência semanal mínima de três vezes na semana, não apresentavam problemas de saúde que causassem impedimentos para a prática do exercício físico e aceitaram participar voluntariamente da pesquisa aceitando o Termo de Consentimento Livro e Esclarecido (TCLE).

\section{Instrumentos}

As variáveis independentes do estudo foram mensuradas a partir de um questionário sociodemográfico, semiestruturado e elaborado pelos próprios autores, averiguando questões referentes a estilo de vida, idade, tempo e tipo de prática, tempo de contaminação do COVID-19, se apresentou desconforto que influenciou no desempenho físico (Huang et al., 2021) ou alguma sintomatologia como dispneia (Iser et al., 2020), fadiga ou fraqueza muscular e redução da massa muscular (Huang et al., 2021).

No mais, através de uma variável dependente, questionário de qualidade de vida SF-36, que é multidimensional, formado por 36 itens, com oito escalas ou componentes: capacidade funcional, aspectos físicos, dor, estado geral de saúde, vitalidade, aspectos sociais, aspectos emocionais e saúde mental. Apresenta uma pontuação/escore final de 0 a 100, no qual zero corresponde ao pior estado geral de saúde e 100 ao melhor estado de saúde (Ciconelli et al., 1999). 


\section{Procedimentos}

Os procedimentos adotados nesta pesquisa estão de acordo com os critérios de ética em pesquisa com seres humanos, baseado na resolução $(466 / 12)$ do Conselho Nacional de Saúde, aprovado pelo Comitê de Ética e Pesquisa da Universidade Federal de Pernambuco (UFPE), CAE: 73905417.0.0000.5208.

Os participantes foram selecionados de forma não-probabilística e por conveniência, a coleta de dados se deu por meio Google formulário. Os sujeitos que tiveram interesse em participar da pesquisa fizeram o aceite do TCLE assinando "concordo". O formulário ficou disponível por 15 dias e divulgado via e-mail e redes sociais (Facebook, Instagram e Whats $A p p$ ), para grupos de academias, praticantes de corrida, ciclismo e outras atividades. Antes do preenchimento, uma breve instrução era dada aos participantes, contendo informações sobre a dificuldade da volta ao exercício relatada pelos pacientes que tiveram COVID-19, aos objetivos da pesquisa, benefícios, contribuições e o tempo gasto para preenchimento do formulário (aproximadamente 10 minutos). Ademais, eram instruídos a responder as perguntas levando em consideração a semana de volta ao exercício físico. No formulário, após o aceite do TCLE, preencheram o questionário sociodemográfico (variáveis dependentes) e, na sequência, foram encaminhados para o preenchimento do questionário SF-36 (Ciconelli, Ferraz, Santos, Meinão, \& Quaresma, 1999).

\section{Análise dos dados}

Os dados não apresentaram normalidade pelo teste de Shapiro-Wilk, nem homogeneidade pelo teste de Levene's. Os valores foram apresentados através da estatística descritiva (média e desvio padrão), categorizado por gênero, além dos valores para os diferentes domínios do questionário SF-36, aplicado no pós COVID-19. $\mathrm{O}$ teste Qui quadrado mostrou associação significativa entre as variáveis fraqueza ou fadiga muscular e dispnéia, com a variável desconforto na volta a prática física. Os dados foram analisados por meio dos softwares SPSS, versão 20.0 (IBM, EUA), considerando nível de significância de $5 \%(\mathrm{p}<0,05)$.

\section{RESULTADOS E DISCUSSÃO}

O estudo foi composto por 34 sujeitos, sendo 20 (58,8\%) mulheres, que apresentavam idade média de $34 \pm 7,5$ anos, praticavam exercício físico regular há 5,8 $\pm 5,9$ anos, tiveram diagnóstico confirmado do COVID-19 há 5,3 $\pm 3,2$ meses, com a grande maioria apresentando sintomas leves $(55,9 \%)$. Sendo que todos os dados caracterizando a amostra estão descritos na tabela 1 . 
Tabela 1. Caracterização da amostra

\begin{tabular}{|c|c|}
\hline & Variável \\
\hline Idade (anos) & $34 \pm 7,5$ \\
\hline Histórico de prática de exercício físico (anos) & $5,8 \pm 5,9$ \\
\hline Há quanto tempo contraiu COVID-19 (meses) & $5,3 \pm 3,2$ \\
\hline Assintomático $\Delta / \mathrm{n}(\%)$ & $3 / 34(8,8)$ \\
\hline Sintomas leves $\Delta / \mathrm{n}(\%)$ & $19 / 34(55,9)$ \\
\hline Sintomas moderados $\Delta / \mathrm{n}(\%)$ & $11 / 34(32,4)$ \\
\hline Sintomas graves $\Delta / \mathrm{n}(\%)$ & $1 / 34(2,9)$ \\
\hline \multicolumn{2}{|c|}{ Homens $(n=14)$} \\
\hline Idade (anos) & $30,7 \pm 7,5$ \\
\hline Histórico de prática de exercício físico (anos) & $7,7 \pm 7,0$ \\
\hline Há quanto tempo contraiu COVID-19 (meses) & $5,0 \pm 2,3$ \\
\hline Assintomático $\Delta / \mathrm{n}(\%)$ & $2 / 14(14,2)$ \\
\hline Sintomas leves $\Delta / \mathrm{n}(\%)$ & $6 / 14(42,9)$ \\
\hline Sintomas moderados $\Delta / \mathrm{n}(\%)$ & $6 / 14(42,9)$ \\
\hline Sintomas graves $\Delta / \mathrm{n}(\%)$ & $0 / 14(0,0)$ \\
\hline \multicolumn{2}{|c|}{ Mulheres $(n=20)$} \\
\hline Idade (anos) & $29,1 \pm 7,9$ \\
\hline Histórico de prática de exercício físico (anos) & $4,5 \pm 4,7$ \\
\hline Há quanto tempo contraiu COVID-19 (meses) & $5,6 \pm 3,7$ \\
\hline Assintomático $\Delta / \mathrm{n}(\%)$ & $1 / 20(5,0)$ \\
\hline Sintomas leves $\Delta / \mathrm{n}(\%)$ & $13 / 20(65,0)$ \\
\hline Sintomas moderados $\Delta / \mathrm{n}(\%)$ & $5 / 20(25,0)$ \\
\hline Sintomas graves $\Delta / \mathrm{n}(\%)$ & $1 / 20(5,0)$ \\
\hline
\end{tabular}

Fonte: elaboração própria

Levando em consideração a semana que voltaram praticar exercício físico após a contaminação do COVID-19, variáveis referentes a qualidade de vida obtidas pelo questionário SF-36 mostraram valores de domínios relativamente menores nas mulheres, destacando capacidade funcional ( 90,7 homens e 77,8 mulheres), limitação dos aspectos emocionais (45,1 homens e 31,4 mulheres), dor (33,6 homens e 36,5 mulheres) e estado geral em saúde (60,4 homens e 54,0 mulheres) (Tabela 2).

Segundo Nguyen e colaboradores (2020) o COVID-19 influencia diretamente na qualidade física da maioria das pessoas que foram acometidas, fato se dá pelo impacto social e emocional da doença, associado a redução dos níveis de atividade física que a fase ativa do vírus ocasiona. No presente estudo podemos observar 
domínios menores do que comparado a outros estudos em pessoas fisicamente ativas (Raafs et al., 2020), evidenciado uma possível influência do COVID-19, sugerindo uma redução das condições de qualidade de vida, mesmo para pessoas fisicamente ativas.

A taxa de mortalidade e complicações com o COVID-19 apresentam valores mais elevados nos homens, devido as mulheres apresentarem respostas imunológicas maiores, quando contaminada, aumentando a atividade inflamatória, podendo estar mais susceptível a sintomatologias agudas após a contaminação (Freire et al., 2020). Fato pode justificar os menores domínios apresentado pelas mulheres, descritos na tabela 2.

Tabela 2. Dados referentes aos diferentes domínios do questionário SF-36, aplicado em pessoas com pós COVID-19

\begin{tabular}{|c|c|c|}
\hline Domínios & $\begin{array}{c}\text { Média } \\
(\text { Total } n=34)\end{array}$ & $\pm \mathbf{d p}$ \\
\hline Capacidade funcional & 83,1 & $\pm 18,2$ \\
\hline Limitação aspectos físicos & 36,8 & $\pm 37,0$ \\
\hline Limitação aspectos emoci onais & 37,1 & $\pm 38,2$ \\
\hline Dor & 35,3 & $\pm 34,5$ \\
\hline Vitalidade & 52,1 & $\pm 12,7$ \\
\hline Saúde mental & 51,5 & $\pm 12,5$ \\
\hline Aspectos sociais & 40,3 & $\pm 23,0$ \\
\hline Estado geral de saúde & 56,6 & $\pm 16,7$ \\
\hline \multicolumn{3}{|c|}{ Homens $(n=14)$} \\
\hline Capacidade funcional & 90,7 & $\pm 15,2$ \\
\hline Limitação aspectos físicos & 38,3 & $\pm 40,1$ \\
\hline Limitação aspectos emoci onais & 45,1 & $\pm 44,6$ \\
\hline Dor & 33,6 & $\pm 32,7$ \\
\hline Vitalidade & 54,6 & $\pm 15,4$ \\
\hline Saúde mental & 53,4 & $\pm 13,1$ \\
\hline Aspectos sociais & 45,3 & \pm 28.8 \\
\hline Estado geral de saúde & 60,4 & $\pm 18,4$ \\
\hline \multicolumn{3}{|c|}{ Mulheres $(n=20)$} \\
\hline Capacidade funcional & 77,8 & $\pm 18,6$ \\
\hline Limitação aspectos físicos & 35,0 & $\pm 35,7$ \\
\hline Limitação aspectos emoci onais & 31,4 & $\pm 33,2$ \\
\hline Dor & 36,5 & $\pm 36,5$ \\
\hline Vitalidade & 50,3 & $\pm 10,6$ \\
\hline Saúde mental & 50,2 & $\pm 12,3$ \\
\hline Aspectos sociais & 36,8 & $\pm 17,9$ \\
\hline Estado geral de saúde & 54,0 & $\pm 15,4$ \\
\hline
\end{tabular}


Quando indagados sobre a sensação do estado de saúde antes do COVID-19 com o estado atual, 6 (17,6\%) sujeitos relataram estar muito melhor, 10 (29,4\%) um pouco melhor, $12(35,3 \%)$ quase a mesma coisa, 6 $(17,6 \%)$ um pouco melhor e nenhum relatou estar muito pior (Tabela 3 ).

A maior parte dos sujeitos (35,3\%) relatam estar quase o mesmo quando comparado ao antes da infecção do COVID-19, fato pode ser justificável pela grande maioria dos sujeitos apresentarem sintomas leves (55,9\%).

As respostas crônicas do exercício físico podem influenciar pela recuperação mais rápida após a infecção, reestabelecendo a saúde de maneira mais rápida (ACSM, 2020). No estudo de Gomes e Paula (2020), podemos observar que a atividade física apresenta relação direta contra os mecanismos fisiopatológicos da COVID-19, tendo potencial para diminuir a gravidade dessa doença, reduzindo a inflamação sistêmica, como também mobilizando as células do sistema imunológico, com aumento da imunovigilância da pessoa pré e pós a infecção, age na prevenção, atenuando os sintomas. Inclusive, a prática regular de exercícios físicos pode melhorar as respostas imunológicas à vacinação, que se encontra atualmente em estágios avançados de testes.

Tabela 3- Dados referentes ao estado atual de saúde dos participantes comparado com antes do diagnóstico da infecção por COVID-19

\begin{tabular}{lc}
\hline $\begin{array}{l}\text { Pergunta: Comparada há antes do } \\
\text { conhecimento da COVID-19, como você }\end{array}$ & $\mathbf{N}^{\circ}$ participantes \\
se classificaria sua saúde em geral, agora? & \\
\hline Muito melhor & 6 \\
Um pouco melhor & 10 \\
Quase a mesma & 6 \\
Um pouco pior & 0 \\
Muito pior & \\
\hline
\end{tabular}

Referente ao desconforto que influenciou no desempenho físico, 22 sujeitos $(64,7 \%$ total; $27,8 \%$ homens e 72,2\% mulheres) relataram que tiveram, sendo 16 das 20 mulheres (80,0\%) e 6 dos 14 homens (42,9\%). Já, referente a sintomatologia 20 (58,8\% total; 35,0\% homens e $65,0 \%$ mulheres) relataram fraqueza ou fadiga muscular, 14 (41,2\%; 35,7\% homens e 64,3\% mulheres) dispneia e 22 (64,7\%; 27,3\% homens e 72,2\% mulheres) redução da massa muscular. Quando associado o desconforto que influenciou no desempenho físico observamos resposta significante com a fraqueza ou fadiga muscular $(\mathrm{p}=0,001)$ e a dispneia $(\mathrm{p}=0,001)$ (Tabela 4).

Mesmo após a forma leve ou assintomática da contaminação pelo COVID-19, o aumento dos níveis inflamatórios de maneira excessiva leva a grande resposta catabólica, aumentando os níveis de fadiga e fraqueza muscular, contribuindo para uma resposta muscular hipotrófica (Huang et al., 2021). Ademais, a infecção tem uma alta afinidade com o sistema respiratório, contribuindo uma inflamação nos alvéolos e, como consequência, levando a quadros dispneicos, acentuado pela resposta catabólica supracitada (Iser et al., 2020). Essas respostas, tem como resultado a redução da tolerância ao esforço, justificando o fato da maioria dos sujeitos apresentarem redução dos níveis de desempenho físico $(64,7 \%)$ e pela sua associação com a fraqueza ou fadiga muscular $(\mathrm{p}=0,001)$ e a dispneia $(\mathrm{p}=0,001)$.

Dessa maneira, um programa de condicionamento físico para os pacientes após a infecção pelo COVID-19 pode ter uma relação direta contra os mecanismos fisiopatológicos da doença, o exercício físico reduz o níel de inflamação sistêmica, mobilizando células do sistema imunológico, aumentando a imunovigilância 
no pós-infecção, agindo como prevenção e atenuação dos sintomas e possíveis sequelas (Gomes e Paula, 2020). Entender as consequências do COVID-19 na retomada ao exercício é de extrema importância para os processos de reabilitação desses pacientes, porém, as suas complicações ainda não estão claras, pela falta de estudo que avaliam o desconforto dos pacientes na retomada da prática, sendo o presente estudo o pioneiro com essa temática.

Tabela 4. Associação do desconforto que influenciou no desempenho físico com as variáveis os sintomas de fraqueza ou fadiga muscular, dispneia e redução da massa muscular

\begin{tabular}{|c|c|c|c|c|}
\hline & \multicolumn{4}{|c|}{ Desconforto que influenciou no desempen ho físico } \\
\hline & $\begin{array}{c}\text { Homens } \\
(\mathrm{n}=14)\end{array}$ & $\begin{array}{c}\text { Feminino } \\
(\mathrm{n}=\mathbf{2 0})\end{array}$ & $\begin{array}{c}\text { Total } \\
(\mathrm{n}=34)\end{array}$ & $\chi^{2}$ \\
\hline Sim & 6 & 16 & 22 & \\
\hline Não & 8 & 4 & 12 & - \\
\hline \multirow[t]{3}{*}{ Total } & 14 & 20 & 34 & \\
\hline & \multicolumn{4}{|c|}{ Fraqueza ou fadiga muscular } \\
\hline & Homens & Mulheres & Total & $P$ \\
\hline Sim & 7 & 13 & 20 & \\
\hline Não & 7 & 7 & 14 & $0,001 *$ \\
\hline \multirow[t]{3}{*}{ Total } & 14 & 20 & 34 & \\
\hline & \multicolumn{4}{|c|}{ Dispneia } \\
\hline & Homens & Mulheres & Total & $P$ \\
\hline $\operatorname{Sim}$ & 5 & 9 & 14 & \\
\hline Não & 9 & 11 & 20 & $0,001 *$ \\
\hline \multirow[t]{3}{*}{ Total } & 14 & 20 & 34 & \\
\hline & \multicolumn{4}{|c|}{ Redução massa muscular } \\
\hline & Homens & Mulheres & Total & $P$ \\
\hline $\operatorname{Sim}$ & 6 & 16 & 22 & \\
\hline Não & 8 & 4 & 12 & 0,104 \\
\hline Total & 14 & 20 & 34 & \\
\hline
\end{tabular}

Fonte: elaboração própria

\section{CONCLUSÕES}

Poucos estudos acercam o exercício físico em relação ao COVID-19, as indicações que temos hoje são referentes a respostas apresentadas com outras infecçóes virais. Contudo, os mecanismos fisiológicos parecem ser semelhantes, proporcionando respostas similares. Tem sido descrito que sequelas estão presente em boa parte dos pacientes que são acometidos pela COVID-19, sendo que após contaminação o exercício físico 
pode auxiliar nesse quadro. Contudo, as mesmas sequelas, podem ser um impedimento na retomada a prática do exercício, devido a dificuldade que esses aspectos fisiopatológicos podem ocasionar.

No presente estudo, observamos que a maior parte dos sujeitos relataram ter desconfortos que impactaram no desempenho físico, especialmente nas mulheres. Sendo que esse a queda do desempenho está ligada a fraqueza ou fadiga muscular e a dispneia. Entretanto, se trata de uma temática pouco explorada, necessitando de mais investigaçóes para evidenciar os possíveis prejuízos que as sequelas decorrentes do COVID-19 podem ocasionar na retomada da prática do exercício físico e suas possíveis consequências.

\section{REFERÊNCIAS}

American College of Sports. (2021). Staying active during the coronavirus pandemic. Recuperado de: https://www.exerciseismedicine.org/assets/page_documents/EIM_Rx\%20for\%20health_\%20Staying\%20 Active\%20During\%20Coronavirus\%20Pandemic.pdf.

Brasil. Ministério da Saúde. (2021). Painel Coronavirus Brasil. Recuperado de: https://covid.saude.gov.br/.

Ciconelli, R. M., Ferraz, M. B., Santos, W. Meinão, I., \& Quaresma, M. R. (1999). Tradução para a língua portuguesa e validação do questionário genérico de avaliação de qualidade de vida SF-36 (Brasil SF-36). Revista Brasileira Reumatologia, São Paulo, 39(3), 143-150.

Gomes, B. B. C. \& Paula, W. F. (2020). Efeitos do exercício físico na prevenção e atenuação da severidade dos sintomas e na reabilitação de individuos infectadospor SARS-COV-2 (Monografia de Graduação em Educação Física, Faculdade de Ciências da Educação e Saúde, Centro Universitário de Brasília, Brasília, DF). Recuperado de: https://repo sitorio.uniceub.br/jspui/handle/prefix/14617

Huang, C., Huang, L., Wang, Y., Li, X., Ren, L,... Cao, B. (2021). 6-month consequences of COVID-19 in patients discharged from hospital: a cohort study. The Lancet, 397(10270), 220-232. https://doi.org/10.1016/S0140-6 736(20)32656-8

Organização Pan-Americana da Saúde (2020). Folha informativa covid-19: escritório da Opas e da OMS no Brasil. Recuperado de: https://bit.ly/3lvKEBl

Freire, P. P., Marques, A. H. C., Baiocchi, G. C., Schimke, L. F., Fonseca, D. L. M.,... Cabral-Marques, O. (2020). Specific immune-regulatory transcriptional signatures reveal sex and age differences in SARS-CoV-2 infected patients. Cold Spring Harbor Laboratory. https://doi.org/10.1101/2020.11.12.20230417

Iser, B. P. M, Sliva, I., Raymundo, V., Poleto, M. B., Schuelter-Trevisol, F., \& Bobinski, F. (2020). Suspected COVID-19 case definition: a narrative review of the most frequent signs and symptoms among confirmed cases. Epidemiol Serv Saude, 29(3), e2020233. http://doi.org/10.5123/S1679-49742020000300018

Luan, X., Tian, X., Zhang, H., Huang, R., Li, N., Chen, P., \& Wang, R. (2019). Exercise as a prescription for patients with various diseases. Journal of Sport and Health Science, 8(5), 422-441. https://doi.org/10.1016/j.jshs.2019 .04 .002

Nguyen, H. C., Nguyen, M. H., Do, B. N., Tran, C. Q., Nguyen, T. T. P.,... Duong, T. V. (2020). People with Suspected COVID-19 Symptoms Were More Likely Depressed and Had Lower Health-Related Quality of Life: The Potential Benefit of Health Literacy.J. Clin. Med. 9(4), 965. https://doi.org/10.3390/jcm9040965

Raafs, B. M. Karssemeijer, E. G. A., Horst, L. V., Aaronson, J. A., Olde, M. G. N. Kessels, R. R. P. C. (2020). Physical Exercise Training Improves Quality of Life in Healthy Older Adults. A Meta-Analysis.J Aging Phys Act. 28(1), 81-93. https://doi.org/10.1123/japa.2018-0436

Sallis, J. \& Pratt, M. (2020). Um apelo à ação: atividade fisica e COVID-19. Exercise is medicine (ACSM). Recuperado de: https://www.exerciseismedicine.org/support_page.php/stories/?b=896

Thomas, J. R., Nelson, J. K., \& Silverman, S. L. (2012). Métodos de pesquisa em atividade física. São Paulo: Ed. 6.

World Health Organization (2021). Coronavirus (COVID-19) Dashboard. Recuperado de: https://covid19.who.int/ 